\title{
Supplementary material for a unified framework for high-dimensional analysis of $M$-estimators with decomposable regularizers
}

\author{
Sahand N. Negahban ${ }^{1}$, Pradeep Ravikumar ${ }^{2}$, \\ Martin J. Wainwright ${ }^{3,4}$ and Bin $\mathbf{Y u}^{3,4}$ \\ MIT, Department of EECS ${ }^{1}$ \\ UT Austin, Department of $\mathrm{CS}^{2}$ \\ UC Berkeley, Department of EECS ${ }^{3,4}$ and Statistics ${ }^{3,4}$
}

In this supplementary text, we include a number of the technical details and proofs for the results presented in the main text.

\section{PROOFS RELATED TO THEOREM 1}

In this section, we collect the proofs of Lemma 1 and our main result. All our arguments in this section are deterministic, and both proofs make use of the function $\mathcal{F}: \mathbb{R}^{p} \rightarrow \mathbb{R}$ given by

$$
\mathcal{F}(\Delta):=\mathcal{L}\left(\theta^{*}+\Delta\right)-\mathcal{L}\left(\theta^{*}\right)+\lambda_{n}\left\{\mathcal{R}\left(\theta^{*}+\Delta\right)-\mathcal{R}\left(\theta^{*}\right)\right\} .
$$

In addition, we exploit the following fact: since $\mathcal{F}(0)=0$, the optimal error $\widehat{\Delta}=\widehat{\theta}-\theta^{*}$ must satisfy $\mathcal{F}(\widehat{\Delta}) \leq 0$.

\subsection{Proof of Lemma 1}

Note that the function $\mathcal{F}$ consists of two parts: a difference of loss functions, and a difference of regularizers. In order to control $\mathcal{F}$, we require bounds on these two quantities:

Sahand Negahban, Department of EECS, Massachusetts Institute of Technology, Cambridge MA 02139 (e-mail: sahandn@mit.edu).Pradeep Ravikumar, Department of CS, University of Texas, Austin, Austin, TX 78701 (e-mail: pradeepr@cs.utexas.edu). Martin J. Wainwright, Department of EECS and Statistics, University of California Berkeley, Berkeley CA 94720 (e-mail: wainwrig@eecs.berkeley.edu). Bin Yu, Department of Statistics, University of California Berkeley, Berkeley CA 94720 (e-mail: binyu@stat.berkeley.edu). Sahand Negahban, Department of EECS, Massachusetts Institute of Technology, Cambridge MA 02139 (e-mail: sahandn@mit.edu).Pradeep Ravikumar, Department of CS, University of Texas, Austin, Austin, TX 78701 (e-mail: pradeepr@cs.utexas.edu). Martin J. Wainwright, Department of EECS and Statistics, University of California Berkeley, Berkeley CA 94720 (e-mail: wainwrig@eecs.berkeley.edu). Bin Yu, Department of Statistics, University of California Berkeley, Berkeley CA94720 (e-mail: binyu@stat.berkeley.edu). 
Lemma 3 (Deviation inequalities). For any decomposable regularizer and $p$ dimensional vectors $\theta^{*}$ and $\Delta$, we have

$$
\mathcal{R}\left(\theta^{*}+\Delta\right)-\mathcal{R}\left(\theta^{*}\right) \geq \mathcal{R}\left(\Delta_{\overline{\mathcal{M}}^{\perp}}\right)-\mathcal{R}\left(\Delta_{\overline{\mathcal{M}}}\right)-2 \mathcal{R}\left(\theta_{\mathcal{M}^{\perp}}^{*}\right) .
$$

Moreover, as long as $\lambda_{n} \geq 2 \mathcal{R}^{*}\left(\nabla \mathcal{L}\left(\theta^{*}\right)\right)$ and $\mathcal{L}$ is convex, we have

$$
\mathcal{L}\left(\theta^{*}+\Delta\right)-\mathcal{L}\left(\theta^{*}\right) \geq-\frac{\lambda_{n}}{2}\left[\mathcal{R}\left(\Delta_{\overline{\mathcal{M}}}\right)+\mathcal{R}\left(\Delta_{\overline{\mathcal{M}}^{\perp}}\right)\right]
$$

ProOF. Since $\mathcal{R}\left(\theta^{*}+\Delta\right)=\mathcal{R}\left(\theta_{\mathcal{M}^{*}}^{*}+\theta_{\mathcal{M}^{\perp}}^{*}+\Delta_{\overline{\mathcal{M}}}+\Delta_{\overline{\mathcal{M}}^{\perp}}\right)$, triangle inequality implies that

$\mathcal{R}\left(\theta^{*}+\Delta\right) \geq \mathcal{R}\left(\theta_{\mathcal{M}}^{*}+\Delta_{\overline{\mathcal{M}}^{\perp}}\right)-\mathcal{R}\left(\theta_{\mathcal{M}^{\perp}}^{*}+\Delta_{\overline{\mathcal{M}}}\right) \geq \mathcal{R}\left(\theta_{\mathcal{M}}^{*}+\Delta_{\overline{\mathcal{M}}^{\perp}}\right)-\mathcal{R}\left(\theta_{\mathcal{M}^{\perp}}^{*}\right)-\mathcal{R}\left(\Delta_{\overline{\mathcal{M}}}\right)$.

By decomposability applied to $\theta_{\mathcal{M}}^{*}$ and $\Delta_{\overline{\mathcal{M}}^{\perp}}$, we have $\mathcal{R}\left(\theta_{\mathcal{M}^{*}}+\Delta_{\overline{\mathcal{M}}^{\perp}}\right)=\mathcal{R}\left(\theta_{\mathcal{M}}^{*}\right)+$ $\mathcal{R}\left(\Delta_{\overline{\mathcal{M}}^{\perp}}\right)$, so that

$$
\mathcal{R}\left(\theta^{*}+\Delta\right) \geq \mathcal{R}\left(\theta_{\mathcal{M}}^{*}\right)+\mathcal{R}\left(\Delta_{\overline{\mathcal{M}}^{\perp}}\right)-\mathcal{R}\left(\theta_{\mathcal{M}^{\perp}}^{*}\right)-\mathcal{R}\left(\Delta_{\overline{\mathcal{M}}}\right)
$$

Similarly, by triangle inequality, we have $\mathcal{R}\left(\theta^{*}\right) \leq \mathcal{R}\left(\theta_{\mathcal{M}}^{*}\right)+\mathcal{R}\left(\theta_{\mathcal{M}^{\perp}}^{*}\right)$. Combining this inequality with the bound (54), we obtain

$$
\begin{aligned}
\mathcal{R}\left(\theta^{*}+\Delta\right)-\mathcal{R}\left(\theta^{*}\right) & \geq \mathcal{R}\left(\theta_{\mathcal{M}}^{*}\right)+\mathcal{R}\left(\Delta_{\overline{\mathcal{M}}^{\perp}}\right)-\mathcal{R}\left(\theta_{\mathcal{M}^{\perp}}^{*}\right)-\mathcal{R}\left(\Delta_{\overline{\mathcal{M}}}\right)-\left\{\mathcal{R}\left(\theta_{\mathcal{M}}^{*}\right)+\mathcal{R}\left(\theta_{\mathcal{M}^{\perp}}^{*}\right)\right\} \\
& =\mathcal{R}\left(\Delta_{\overline{\mathcal{M}}^{\perp}}\right)-\mathcal{R}\left(\Delta_{\overline{\mathcal{M}}}\right)-2 \mathcal{R}\left(\theta_{\mathcal{M}^{\perp}}^{*}\right)
\end{aligned}
$$

which yields the claim (52).

Turning to the loss difference, using the convexity of the loss function $\mathcal{L}$, we have

$$
\mathcal{L}\left(\theta^{*}+\Delta\right)-\mathcal{L}\left(\theta^{*}\right) \geq\left\langle\nabla \mathcal{L}\left(\theta^{*}\right), \Delta\right\rangle \geq-\left|\left\langle\nabla \mathcal{L}\left(\theta^{*}\right), \Delta\right\rangle\right| .
$$

Applying the (generalized) Cauchy-Schwarz inequality with the regularizer and its dual, we obtain

$$
\left|\left\langle\nabla \mathcal{L}\left(\theta^{*}\right), \Delta\right\rangle\right| \leq \mathcal{R}^{*}\left(\nabla \mathcal{L}\left(\theta^{*}\right)\right) \mathcal{R}(\Delta) \leq \frac{\lambda_{n}}{2}\left[\mathcal{R}\left(\Delta_{\overline{\mathcal{M}}}\right)+\mathcal{R}\left(\Delta_{\overline{\mathcal{M}}^{\perp}}\right)\right]
$$

where the final equality uses triangle inequality, and the assumed bound $\lambda_{n} \geq 2 \mathcal{R}^{*}\left(\nabla \mathcal{L}\left(\theta^{*}\right)\right)$. Consequently, we conclude that

$$
\mathcal{L}\left(\theta^{*}+\Delta\right)-\mathcal{L}\left(\theta^{*}\right) \geq-\frac{\lambda_{n}}{2}\left[\mathcal{R}\left(\Delta_{\overline{\mathcal{M}}}\right)+\mathcal{R}\left(\Delta_{\overline{\mathcal{M}}^{\perp}}\right)\right]
$$

as claimed.

We can now complete the proof of Lemma 1. Combining the two lower bounds (52) and (53), we obtain

$$
\begin{aligned}
0 \geq \mathcal{F}(\widehat{\Delta}) & \geq \lambda_{n}\left\{\mathcal{R}\left(\Delta_{\overline{\mathcal{M}}^{\perp}}\right)-\mathcal{R}\left(\Delta_{\overline{\mathcal{M}}}\right)-2 \mathcal{R}\left(\theta_{\mathcal{M}^{\perp}}^{*}\right)\right\}-\frac{\lambda_{n}}{2}\left[\mathcal{R}\left(\Delta_{\overline{\mathcal{M}}}\right)+\mathcal{R}\left(\Delta_{\overline{\mathcal{M}}^{\perp}}\right)\right] \\
& =\frac{\lambda_{n}}{2}\left\{\mathcal{R}\left(\Delta_{\overline{\mathcal{M}}^{\perp}}\right)-3 \mathcal{R}\left(\Delta_{\overline{\mathcal{M}}}\right)-4 \mathcal{R}\left(\theta_{\mathcal{M}^{\perp}}^{*}\right)\right\}
\end{aligned}
$$

from which the claim follows. 


\subsection{Proof of Theorem 1}

Recall the set $\mathbb{C}\left(\mathcal{M}, \overline{\mathcal{M}}^{\perp} ; \theta^{*}\right)$ from equation (17). Since the subspace pair $\left(\mathcal{M}, \overline{\mathcal{M}}^{\perp}\right)$ and true parameter $\theta^{*}$ remain fixed throughout this proof, we adopt the shorthand notation $\mathbb{C}$. Letting $\delta>0$ be a given error radius, the following lemma shows that it suffices to control the sign of the function $\mathcal{F}$ over the set $\mathbb{K}(\delta):=\mathbb{C} \cap\{\|\Delta\|=\delta\}$.

LEMmA 4. If $\mathcal{F}(\Delta)>0$ for all vectors $\Delta \in \mathbb{K}(\delta)$, then $\|\widehat{\Delta}\| \leq \delta$.

Proof. We first claim that $\mathbb{C}$ is star-shaped, meaning that if $\widehat{\Delta} \in \mathbb{C}$, then the entire line $\{t \widehat{\Delta} \mid t \in(0,1)\}$ connecting $\widehat{\Delta}$ with the all-zeroes vector is contained with $\mathbb{C}$. This property is immediate whenever $\theta^{*} \in \mathcal{M}$, since $\mathbb{C}$ is then a cone, as illustrated in Figure 1(a). Now consider the general case, when $\theta^{*} \notin \mathcal{M}$. We first observe that for any $t \in(0,1)$,

$$
\Pi_{\overline{\mathcal{M}}}(t \Delta)=\arg \min _{\gamma \in \overline{\mathcal{M}}}\|t \Delta-\gamma\|=t \arg \min _{\gamma \in \overline{\mathcal{M}}}\left\|\Delta-\frac{\gamma}{t}\right\|=t \Pi_{\overline{\mathcal{M}}}(\Delta),
$$

using the fact that $\gamma / t$ also belongs to the subspace $\overline{\mathcal{M}}$. A similar argument can be used to establish the equality $\Pi_{\overline{\mathcal{M}}^{\perp}}(t \Delta)=t \Pi_{\overline{\mathcal{M}}^{\perp}}(\Delta)$. Consequently, for all $\Delta \in \mathbb{C}$, we have

$$
\begin{aligned}
\mathcal{R}\left(\Pi_{\overline{\mathcal{M}}^{\perp}}(t \Delta)\right)=\mathcal{R}\left(t \Pi_{\overline{\mathcal{M}}^{\perp}}(\Delta)\right) & \stackrel{(i)}{=} t \mathcal{R}\left(\Pi_{\overline{\mathcal{M}}^{\perp}}(\Delta)\right) \\
& \stackrel{(i i)}{\leq} t\left\{3 \mathcal{R}\left(\Pi_{\overline{\mathcal{M}}}(\Delta)\right)+4 \mathcal{R}\left(\Pi_{\mathcal{M}^{\perp}}\left(\theta^{*}\right)\right)\right\}
\end{aligned}
$$

where step (i) uses the fact that any norm is positive homogeneous, ${ }^{1}$ and step (ii) uses the inclusion $\Delta \in \mathbb{C}$. We now observe that $3 t \mathcal{R}\left(\Pi_{\mathcal{M}}(\Delta)\right)=3 \mathcal{R}\left(\Pi_{\overline{\mathcal{M}}}(t \Delta)\right)$, and moreover, since $t \in(0,1)$, we have $4 t \mathcal{R}\left(\Pi_{\mathcal{M}^{\perp}}\left(\theta^{*}\right)\right) \leq 4 \mathcal{R}\left(\Pi_{\mathcal{M}^{\perp}}\left(\theta^{*}\right)\right)$. Putting together the pieces, we find that

$$
\begin{aligned}
\mathcal{R}\left(\Pi_{\overline{\mathcal{M}}^{\perp}}(t \Delta)\right) & \leq 3 \mathcal{R}\left(\Pi_{\overline{\mathcal{M}}}(t \Delta)\right)+t 4 \Pi_{\mathcal{M}^{\perp}}\left(\theta^{*}\right) \\
& \leq 3 \mathcal{R}\left(\Pi_{\overline{\mathcal{M}}}(t \Delta)\right)+4 \mathcal{R}\left(\Pi_{\mathcal{M}^{\perp}}\left(\theta^{*}\right)\right),
\end{aligned}
$$

showing that $t \Delta \in \mathbb{C}$ for all $t \in(0,1)$, and hence that $\mathbb{C}$ is star-shaped.

Turning to the lemma itself, we prove the contrapositive statement: in particular, we show that if for some optimal solution $\widehat{\theta}$, the associated error vector $\widehat{\Delta}=\widehat{\theta}-\theta^{*}$ satisfies the inequality $\|\widehat{\Delta}\|>\delta$, then there must be some vector $\widetilde{\Delta} \in \mathbb{K}(\delta)$ such that $\mathcal{F}(\widetilde{\Delta}) \leq 0$. If $\|\widehat{\Delta}\|>\delta$, then the line joining $\widehat{\Delta}$ to 0 must intersect the set $\mathbb{K}(\delta)$ at some intermediate point $t^{*} \widehat{\Delta}$, for some $t^{*} \in(0,1)$. Since the loss function $\mathcal{L}$ and regularizer $\mathcal{R}$ are convex, the function $\mathcal{F}$ is also convex for any choice of the regularization parameter, so that by Jensen's inequality,

$$
\mathcal{F}\left(t^{*} \Delta\right)=\mathcal{F}\left(t^{*} \Delta+\left(1-t^{*}\right) 0\right) \leq t^{*} \mathcal{F}(\widehat{\Delta})+\left(1-t^{*}\right) \mathcal{F}(0) \stackrel{(i)}{=} t^{*} \mathcal{F}(\widehat{\Delta}),
$$

where equality (i) uses the fact that $\mathcal{F}(0)=0$ by construction. But since $\widehat{\Delta}$ is optimal, we must have $\mathcal{F}(\widehat{\Delta}) \leq 0$, and hence $\mathcal{F}\left(t^{*} \Delta\right) \leq 0$ as well. Thus, we have constructed a vector $\widetilde{\Delta}=t^{*} \Delta$ with the claimed properties, thereby establishing Lemma 4.

\footnotetext{
${ }^{1}$ Explicitly, for any norm and non-negative scalar $t$, we have $\|t x\|=t\|x\|$.
} 
On the basis of Lemma 4, the proof of Theorem 1 will be complete if we can establish a lower bound on $\mathcal{F}(\Delta)$ over $\mathbb{K}(\delta)$ for an appropriately chosen radius $\delta>0$. For an arbitrary $\Delta \in \mathbb{K}(\delta)$, we have

$$
\begin{aligned}
\mathcal{F}(\Delta) & =\mathcal{L}\left(\theta^{*}+\Delta\right)-\mathcal{L}\left(\theta^{*}\right)+\lambda_{n}\left\{\mathcal{R}\left(\theta^{*}+\Delta\right)-\mathcal{R}\left(\theta^{*}\right)\right\} \\
& \stackrel{(i)}{\geq}\left\langle\nabla \mathcal{L}\left(\theta^{*}\right), \Delta\right\rangle+\kappa_{\mathcal{L}}\|\Delta\|^{2}-\tau_{\mathcal{L}}^{2}\left(\theta^{*}\right)+\lambda_{n}\left\{\mathcal{R}\left(\theta^{*}+\Delta\right)-\mathcal{R}\left(\theta^{*}\right)\right\} \\
& \stackrel{(i i)}{\geq}\left\langle\nabla \mathcal{L}\left(\theta^{*}\right), \Delta\right\rangle+\kappa_{\mathcal{L}}\|\Delta\|^{2}-\tau_{\mathcal{L}}^{2}\left(\theta^{*}\right)+\lambda_{n}\left\{\mathcal{R}\left(\Delta_{\overline{\mathcal{M}}^{\perp}}\right)-\mathcal{R}\left(\Delta_{\overline{\mathcal{M}}}\right)-2 \mathcal{R}\left(\theta_{\mathcal{M}^{\perp}}^{*}\right)\right\},
\end{aligned}
$$

where inequality (i) follows from the RSC condition, and inequality (ii) follows from the bound (52).

By the Cauchy-Schwarz inequality applied to the regularizer $\mathcal{R}$ and its dual $\mathcal{R}^{*}$, we have $\left|\left\langle\nabla \mathcal{L}\left(\theta^{*}\right), \Delta\right\rangle\right| \leq \mathcal{R}^{*}\left(\nabla \mathcal{L}\left(\theta^{*}\right)\right) \mathcal{R}(\Delta)$. Since $\lambda_{n} \geq 2 \mathcal{R}^{*}\left(\nabla \mathcal{L}\left(\theta^{*}\right)\right)$ by assumption, we conclude that $\left|\left\langle\nabla \mathcal{L}\left(\theta^{*}\right), \Delta\right\rangle\right| \leq \frac{\lambda_{n}}{2} \mathcal{R}(\Delta)$, and hence that

$$
\mathcal{F}(\Delta) \geq \kappa_{\mathcal{L}}\|\Delta\|^{2}-\tau_{\mathcal{L}}^{2}\left(\theta^{*}\right)+\lambda_{n}\left\{\mathcal{R}\left(\Delta_{\overline{\mathcal{M}}^{\perp}}\right)-\mathcal{R}\left(\Delta_{\overline{\mathcal{M}}}\right)-2 \mathcal{R}\left(\theta_{\mathcal{M}^{\perp}}^{*}\right)\right\}-\frac{\lambda_{n}}{2} \mathcal{R}(\Delta)
$$

By triangle inequality, we have $\mathcal{R}(\Delta)=\mathcal{R}\left(\Delta_{\overline{\mathcal{M}}^{\perp}}+\Delta_{\overline{\mathcal{M}}}\right) \leq \mathcal{R}\left(\Delta_{\overline{\mathcal{M}}^{\perp}}\right)+\mathcal{R}\left(\Delta_{\overline{\mathcal{M}}}\right)$, and hence, following some algebra

$$
\begin{aligned}
\mathcal{F}(\Delta) & \geq \kappa_{\mathcal{L}}\|\Delta\|^{2}-\tau_{\mathcal{L}}^{2}\left(\theta^{*}\right)+\lambda_{n}\left\{\frac{1}{2} \mathcal{R}\left(\Delta_{\overline{\mathcal{M}}^{\perp}}\right)-\frac{3}{2} \mathcal{R}\left(\Delta_{\overline{\mathcal{M}}}\right)-2 \mathcal{R}\left(\theta_{\mathcal{M}^{\perp}}^{*}\right)\right\} \\
& \geq \kappa_{\mathcal{L}}\|\Delta\|^{2}-\tau_{\mathcal{L}}^{2}\left(\theta^{*}\right)-\frac{\lambda_{n}}{2}\left\{3 \mathcal{R}\left(\Delta_{\overline{\mathcal{M}}}\right)+4 \mathcal{R}\left(\theta_{\mathcal{M}^{\perp}}^{*}\right)\right\} .
\end{aligned}
$$

Now by definition (21) of the subspace compatibility, we have the inequality $\mathcal{R}\left(\Delta_{\overline{\mathcal{M}}}\right) \leq \Psi(\overline{\mathcal{M}})\left\|\Delta_{\overline{\mathcal{M}}}\right\|$. Since the projection $\Delta_{\overline{\mathcal{M}}}=\Pi_{\overline{\mathcal{M}}}(\Delta)$ is defined in terms of the norm $\|\cdot\|$, it is non-expansive. Since $0 \in \overline{\mathcal{M}}$, we have

$$
\left\|\Delta_{\overline{\mathcal{M}}}\right\|=\left\|\Pi_{\overline{\mathcal{M}}}(\Delta)-\Pi_{\overline{\mathcal{M}}}(0)\right\| \stackrel{(i)}{\leq}\|\Delta-0\|=\|\Delta\|,
$$

where inequality (i) uses non-expansivity of the projection. Combining with the earlier bound, we conclude that $\mathcal{R}\left(\Delta_{\overline{\mathcal{M}}}\right) \leq \Psi(\overline{\mathcal{M}})\|\Delta\|$. Substituting into the lower bound (55), we obtain $\mathcal{F}(\Delta) \geq \kappa_{\mathcal{L}}\|\Delta\|^{2}-\tau_{\mathcal{L}}^{2}\left(\theta^{*}\right)-\frac{\lambda_{n}}{2}\left\{3 \Psi(\overline{\mathcal{M}})\|\Delta\|+4 \mathcal{R}\left(\theta_{\mathcal{M}^{\perp}}^{*}\right)\right\}$. The right-hand side of this inequality is a strictly positive definite quadratic form in $\|\Delta\|$, and so will be positive for $\|\Delta\|$ sufficiently large. In particular, some algebra shows that this is the case as long as

$$
\|\Delta\|^{2} \geq \delta^{2}:=9 \frac{\lambda_{n}^{2}}{\kappa_{\mathcal{L}}^{2}} \Psi^{2}(\overline{\mathcal{M}})+\frac{\lambda_{n}}{\kappa_{\mathcal{L}}}\left\{2 \tau_{\mathcal{L}}^{2}\left(\theta^{*}\right)+4 \mathcal{R}\left(\theta_{\mathcal{M}^{\perp}}^{*}\right)\right\}
$$

thereby completing the proof of Theorem 1.

\section{PROOF OF LEMMA 2}

For any $\Delta$ in the set $\mathbb{C}\left(S_{\eta}\right)$, we have

$$
\begin{aligned}
\|\Delta\|_{1} & \leq 4\left\|\Delta_{S_{\eta}}\right\|_{1}+4\left\|\theta_{S_{\eta}^{c}}^{*}\right\|_{1} \leq \sqrt{\left|S_{\eta}\right|}\|\Delta\|_{2}+4 R_{q} \eta^{1-q} \\
& \leq 4 \sqrt{R_{q}} \eta^{-q / 2}\|\Delta\|_{2}+4 R_{q} \eta^{1-q}
\end{aligned}
$$

imsart-sts ver. 2012/04/10 file: B_BaseFileStatSci.tex date: May 25, 2012 
where we have used the bounds (39) and (40). Therefore, for any vector $\Delta \in \mathbb{C}\left(S_{\eta}\right)$, the condition (31) implies that

$$
\begin{aligned}
\frac{\|X \Delta\|_{2}}{\sqrt{n}} & \geq \kappa_{1}\|\Delta\|_{2}-\kappa_{2} \sqrt{\frac{\log p}{n}}\left\{\sqrt{R_{q}} \eta^{-q / 2}\|\Delta\|_{2}+R_{q} \eta^{1-q}\right\} \\
& =\|\Delta\|_{2}\left\{\kappa_{1}-\kappa_{2} \sqrt{\frac{R_{q} \log p}{n}} \eta^{-q / 2}\right\}-\kappa_{2} \sqrt{\frac{\log p}{n}} R_{q} \eta^{1-q} .
\end{aligned}
$$

By our choices $\eta=\frac{\lambda_{n}}{\kappa_{1}}$ and $\lambda_{n}=4 \sigma \sqrt{\frac{\log p}{n}}$, we have

$$
\kappa_{2} \sqrt{\frac{R_{q} \log p}{n}} \eta^{-q / 2}=\frac{\kappa_{2}}{(8 \sigma)^{q / 2}} \sqrt{R_{q}}\left(\frac{\log p}{n}\right)^{1-\frac{q}{2}},
$$

which is less than $\kappa_{1} / 2$ under the stated assumptions. Thus, we obtain the lower bound

$$
\frac{\|X \Delta\|_{2}}{\sqrt{n}} \geq \frac{\kappa_{1}}{2}\|\Delta\|_{2}-2 \kappa_{2} \sqrt{\frac{\log p}{n}} R_{q} \eta^{1-q},
$$

as claimed.

\section{PROOFS FOR GROUP-SPARSE NORMS}

In this section, we collect the proofs of results related to the group-sparse norms in Section 5.

\subsection{Proof of Proposition 1}

The proof of this result follows similar lines to the proof of condition (31) given by Raskutti et al. [58], hereafter RWY, who established this result in the special case of the $\ell_{1}$-norm. Here we describe only those portions of the proof that require modification. For a radius $t>0$, define the set

$$
V(t):=\left\{\theta \in \mathbb{R}^{p} \mid\left\|\Sigma^{1 / 2} \theta\right\|_{2}=1,\|\theta\|_{\mathcal{G}, \alpha} \leq t\right\},
$$

as well as the random variable $M(t ; X):=1-\inf _{\theta \in V(t)} \frac{\|X \theta\|_{2}}{\sqrt{n}}$. The argument in Section 4.2 of RWY makes use of the Gordon-Slepian comparison inequality in order to upper bound this quantity. Following the same steps, we obtain the modified upper bound

$$
\mathbb{E}[M(t ; X)] \leq \frac{1}{4}+\frac{1}{\sqrt{n}} \mathbb{E}\left[\max _{j=1, \ldots, N_{\mathcal{G}}}\left\|w_{G_{j}}\right\|_{\alpha^{*}}\right] t,
$$

where $w \sim N(0, \Sigma)$. The argument in Section 4.3 uses concentration of measure to show that this same bound will hold with high probability for $M(t ; X)$ itself; the same reasoning applies here. Finally, the argument in Section 4.4 of RWY uses a peeling argument to make the bound suitably uniform over choices of the radius $t$. This argument allows us to conclude that

$$
\begin{aligned}
& \inf _{\theta \in \mathbb{R}^{p}} \frac{\|X \theta\|_{2}}{\sqrt{n}} \geq \frac{1}{4}\left\|\Sigma^{1 / 2} \theta\right\|_{2}-9 \mathbb{E}\left[\max _{j=1, \ldots, N_{\mathcal{G}}}\left\|w_{G_{j}}\right\| \alpha^{*}\right]\|\theta\|_{\mathcal{G}, \alpha} \text { for all } \theta \in \mathbb{R}^{p} \\
& \text { imsart-sts ver. } 2012 / 04 / 10 \text { file: B_BaseFileStatSci.tex date: May 25, } 2012
\end{aligned}
$$


with probability greater than $1-c_{1} \exp \left(-c_{2} n\right)$. Recalling the definition of $\rho_{\mathcal{G}}\left(\alpha^{*}\right)$, we see that in the case $\Sigma=I_{p \times p}$, the claim holds with constants $\left(\kappa_{1}, \kappa_{2}\right)=\left(\frac{1}{4}, 9\right)$. Turning to the case of general $\Sigma$, let us define the matrix norm

$$
\|A\|_{\alpha^{*}}:=\max _{\|\beta\|_{\alpha^{*}=1}}\|A \beta\|_{\alpha^{*}}
$$

With this notation, some algebra shows that the claim holds with

$$
\kappa_{1}=\frac{1}{4} \lambda_{\min }\left(\Sigma^{1 / 2}\right), \quad \text { and } \quad \kappa_{2}=9 \max _{t=1, \ldots, N_{\mathcal{G}}}\left\|\left(\Sigma^{1 / 2}\right)_{G_{t}}\right\|_{\alpha^{*}} .
$$

\subsection{Proof of Corollary 4}

In order to prove this claim, we need to verify that Theorem 1 may be applied. Doing so requires defining the appropriate model and perturbation subspaces, computing the compatibility constant, and checking that the specified choice (48) of regularization parameter $\lambda_{n}$ is valid. For a given subset $S_{\mathcal{G}} \subseteq\left\{1,2, \ldots, N_{\mathcal{G}}\right\}$, define the subspaces

$$
\begin{aligned}
& \mathcal{M}\left(S_{\mathcal{G}}\right):=\left\{\theta \in \mathbb{R}^{p} \mid \theta_{G_{t}}=0 \quad \text { for all } t \notin S_{\mathcal{G}}\right\}, \quad \text { and } \\
& \mathcal{M}^{\perp}\left(S_{\mathcal{G}}\right):=\left\{\theta \in \mathbb{R}^{p} \mid \theta_{G_{t}}=0 \text { for all } t \in S_{\mathcal{G}}\right\} .
\end{aligned}
$$

As discussed in Example 2, the block norm $\|\cdot\|_{\mathcal{G}, \alpha}$ is decomposable with respect to these subspaces. Let us compute the regularizer-error compatibility function, as defined in equation $(21)$, that relates the regularizer $\left(\|\cdot\|_{\mathcal{G}, \alpha}\right.$ in this case) to the error norm (here the $\ell_{2}$-norm). For any $\Delta \in \mathcal{M}\left(S_{\mathcal{G}}\right)$, we have

$$
\|\Delta\|_{\mathcal{G}, \alpha}=\sum_{t \in S_{\mathcal{G}}}\left\|\Delta_{G_{t}}\right\|_{\alpha} \stackrel{(a)}{\leq} \sum_{t \in S_{\mathcal{G}}}\left\|\Delta_{G_{t}}\right\|_{2} \leq \sqrt{s}\|\Delta\|_{2}
$$

where inequality (a) uses the fact that $\alpha \geq 2$.

Finally, let us check that the specified choice of $\lambda_{n}$ satisfies the condition (23). As in the proof of Corollary 2 , we have $\nabla \mathcal{L}\left(\theta^{*} ; Z_{1}^{n}\right)=\frac{1}{n} X^{T} w$, so that the final step is to compute an upper bound on the quantity

$$
\mathcal{R}^{*}\left(\frac{1}{n} X^{T} w\right)=\max _{t=1, \ldots, N_{\mathcal{G}}}\left\|\frac{1}{n}\left(X^{T} w\right)_{G_{t}}\right\|_{\alpha^{*}}
$$

that holds with high probability.

Lemma 5. Suppose that $X$ satisfies the block column normalization condition, and the observation noise is sub-Gaussian (33). Then we have

$$
\mathbb{P}\left[\max _{t=1, \ldots, N_{\mathcal{G}}}\left\|\frac{X_{G_{t}}^{T} w}{n}\right\|_{\alpha^{*}} \geq 2 \sigma\left\{\frac{m^{1-1 / \alpha}}{\sqrt{n}}+\sqrt{\frac{\log N_{\mathcal{G}}}{n}}\right\}\right] \leq 2 \exp \left(-2 \log N_{\mathcal{G}}\right)
$$

Proof. Throughout the proof, we assume without loss of generality that $\sigma=1$, since the general result can be obtained by rescaling. For a fixed group $G$ of size $m$, consider the submatrix $X_{G} \in \mathbb{R}^{n \times m}$. We begin by establishing a tail bound for the random variable $\left\|\frac{X_{G}^{T} w}{n}\right\|_{\alpha^{*}}$. 
Deviations above the mean: For any pair $w, w^{\prime} \in \mathbb{R}^{n}$, we have

$$
\left|\left\|\frac{X_{G}^{T} w}{n}\right\|_{\alpha^{*}}-\left\|\frac{X_{G}^{T} w^{\prime}}{n}\right\|_{\alpha^{*}}\right| \leq \frac{1}{n}\left\|X_{G}^{T}\left(w-w^{\prime}\right)\right\|_{\alpha^{*}}=\frac{1}{n} \max _{\|\theta\|_{\alpha}=1}\left\langle X_{G} \theta,\left(w-w^{\prime}\right)\right\rangle .
$$

By definition of the $(\alpha \rightarrow 2)$ operator norm, we have

$$
\frac{1}{n}\left\|X_{G}^{T}\left(w-w^{\prime}\right)\right\|_{\alpha^{*}} \leq \frac{1}{n}\left\|X_{G}\right\|_{\alpha \rightarrow 2}\left\|w-w^{\prime}\right\|_{2} \stackrel{(i)}{\leq} \frac{1}{\sqrt{n}}\left\|w-w^{\prime}\right\|_{2},
$$

where inequality (i) uses the block normalization condition (47). We conclude that the function $w \mapsto\left\|\frac{X_{G}^{T} w}{n}\right\|_{\alpha^{*}}$ is a Lipschitz with constant $1 / \sqrt{n}$, so that by Gaussian concentration of measure for Lipschitz functions [39], we have

$$
\mathbb{P}\left[\left\|\frac{X_{G}^{T} w}{n}\right\|_{\alpha^{*}} \geq \mathbb{E}\left[\left\|\frac{X_{G}^{T} w}{n}\right\|_{\alpha^{*}}\right]+\delta\right] \leq 2 \exp \left(-\frac{n \delta^{2}}{2}\right) \quad \text { for all } \delta>0 .
$$

Upper bounding the mean: For any vector $\beta \in \mathbb{R}^{m}$, define the zero-mean Gaussian random variable $Z_{\beta}=\frac{1}{n}\left\langle\beta, X_{G}^{T} w\right\rangle$, and note the relation $\left\|\frac{X_{G}^{T} w}{n}\right\|_{\alpha^{*}}=\max _{\|\beta\|_{\alpha}=1} Z_{\beta}$. Thus, the quantity of interest is the supremum of a Gaussian process, and can be upper bounded using Gaussian comparison principles. For any two vectors $\|\beta\|_{\alpha} \leq 1$ and $\left\|\beta^{\prime}\right\|_{\alpha} \leq 1$, we have

$$
\begin{aligned}
\mathbb{E}\left[\left(Z_{\beta}-Z_{\beta^{\prime}}\right)^{2}\right] & =\frac{1}{n^{2}}\left\|X_{G}\left(\beta-\beta^{\prime}\right)\right\|_{2}^{2} \\
& \stackrel{(a)}{\leq} \frac{2}{n} \frac{\left\|X_{G}\right\|_{\alpha \rightarrow 2}^{2}}{n}\left\|\beta-\beta^{\prime}\right\|_{2}^{2} \\
& \stackrel{(b)}{\leq} \frac{2}{n}\left\|\beta-\beta^{\prime}\right\|_{2}^{2},
\end{aligned}
$$

where inequality (a) uses the fact that $\left\|\beta-\beta^{\prime}\right\|_{\alpha} \leq \sqrt{2}$, and inequality (b) uses the block normalization condition (47).

Now define a second Gaussian process $Y_{\beta}=\sqrt{\frac{2}{n}}\langle\beta, \varepsilon\rangle$, where $\varepsilon \sim N\left(0, I_{m \times m}\right)$ is standard Gaussian. By construction, for any pair $\beta, \beta^{\prime} \in \mathbb{R}^{m}$, we have

$$
\mathbb{E}\left[\left(Y_{\beta}-Y_{\beta^{\prime}}\right)^{2}\right]=\frac{2}{n}\left\|\beta-\beta^{\prime}\right\|_{2}^{2} \geq \mathbb{E}\left[\left(Z_{\beta}-Z_{\beta^{\prime}}\right)^{2}\right],
$$

so that the Sudakov-Fernique comparison principle [39] implies that

$$
\mathbb{E}\left[\left\|\frac{X_{G}^{T} w}{n}\right\|_{\alpha^{*}}\right]=\mathbb{E}\left[\max _{\|\beta\|_{\alpha}=1} Z_{\beta}\right] \leq \mathbb{E}\left[\max _{\|\beta\|_{\alpha}=1} Y_{\beta}\right] .
$$

By definition of $Y_{\beta}$, we have

$$
\begin{aligned}
\mathbb{E}\left[\max _{\|\beta\|_{\alpha}=1} Y_{\beta}\right]=\sqrt{\frac{2}{n}} \mathbb{E}\left[\|\varepsilon\|_{\alpha^{*}}\right] & =\sqrt{\frac{2}{n}} \mathbb{E}\left[\left(\sum_{j=1}^{m}\left|\varepsilon_{j}\right|^{\alpha^{*}}\right)^{1 / \alpha^{*}}\right] \\
& \leq \sqrt{\frac{2}{n}} m^{1 / \alpha^{*}}\left(\mathbb{E}\left[\left|\varepsilon_{1}\right|^{\alpha^{*}}\right]\right)^{1 / \alpha^{*}}
\end{aligned}
$$


using Jensen's inequality, and the concavity of the function $f(t)=t^{1 / \alpha^{*}}$ for $\alpha^{*} \in[1,2]$. Finally, we have

$$
\left(\mathbb{E}\left[\left|\varepsilon_{1}\right|^{\alpha^{*}}\right]\right)^{1 / \alpha^{*}} \leq \sqrt{\mathbb{E}\left[\varepsilon_{1}^{2}\right]}=1,
$$

and $1 / \alpha^{*}=1-1 / \alpha$, so that we have shown that

$$
\mathbb{E}\left[\max _{\|\beta\|_{\alpha}=1} Y_{\beta}\right] \leq 2 \frac{m^{1-1 / \alpha}}{\sqrt{n}}
$$

Combining this bound with the concentration statement (57), we obtain

$$
\mathbb{P}\left[\left\|\frac{X_{G}^{T} w}{n}\right\|_{\alpha^{*}} \geq 2 \frac{m^{1-1 / \alpha}}{\sqrt{n}}+\delta\right] \leq 2 \exp \left(-\frac{n \delta^{2}}{2}\right) .
$$

We now apply the union bound over all groups, and set $\delta^{2}=\frac{4 \log N_{\mathcal{G}}}{n}$ to conclude that

$$
\mathbb{P}\left[\max _{t=1, \ldots, N_{\mathcal{G}}}\left\|\frac{X_{G_{t}}^{T} w}{n}\right\|_{\alpha^{*}} \geq 2\left\{\frac{m^{1-1 / \alpha}}{\sqrt{n}}+\sqrt{\frac{\log N_{\mathcal{G}}}{n}}\right\}\right] \leq 2 \exp \left(-2 \log N_{\mathcal{G}}\right),
$$

as claimed. 


\section{REFERENCES}

[1] Large Synoptic Survey Telescope, 2003. URL: www.1sst.org.

[2] A. Agarwal, S. Negahban, and M. J. Wainwright. Noisy matrix decomposition via convex relaxation: Optimal rates in high dimensions. To appear in Annals of Statistics, 2011. Appeared as http://arxiv.org/abs/1102.4807.

[3] F. Bach. Consistency of the group Lasso and multiple kernel learning. Journal of Machine Learning Research, 9:1179-1225, 2008.

[4] F. Bach. Consistency of trace norm minimization. Journal of Machine Learning Research, 9:1019-1048, June 2008.

[5] F. Bach. Self-concordant analysis for logistic regression. Electronic Journal of Statistics, 4:384-414, 2010.

[6] R. G. Baraniuk, V. Cevher, M. F. Duarte, and C. Hegde. Model-based compressive sensing. Technical report, Rice University, 2008. Available at arxiv:0808.3572.

[7] P. J. Bickel, J. B. Brown, H. Huang, and Q. Li. An overview of recent developments in genomics and associated statistical methods. Phil. Trans. Royal Society A, 367:4313-4337, 2009.

[8] P. J. Bickel and E. Levina. Covariance regularization by thresholding. Annals of Statistics, 36(6):2577-2604, 2008.

[9] P. J. Bickel, Y. Ritov, and A. Tsybakov. Simultaneous analysis of Lasso and Dantzig selector. Annals of Statistics, 37(4):1705-1732, 2009.

[10] F. Bunea. Honest variable selection in linear and logistic regression models via $\ell_{1}$ and $\ell_{1}+\ell_{2}$ penalization. Electronic Journal of Statistics, 2:1153-1194, 2008.

[11] F. Bunea, Y. She, and M. Wegkamp. Adaptive rank penalized estimators in multivariate regression. Technical report, Florida State, 2010. available at arXiv:1004.2995.

[12] F. Bunea, A. Tsybakov, and M. Wegkamp. Aggregation for gaussian regression. Annals of Statistics, 35(4):1674-1697, 2007.

[13] F. Bunea, A. Tsybakov, and M. Wegkamp. Sparsity oracle inequalities for the Lasso. Electronic Journal of Statistics, pages 169-194, 2007.

[14] T. Cai and H. Zhou. Optimal rates of convergence for sparse covariance matrix estimation. Technical report, Wharton School of Business, University of Pennsylvania, 2010. available at http://www-stat.wharton.upenn.edu/tcai/paper/html/Sparse-Covariance-Matrix.html.

[15] E. Candes and T. Tao. Decoding by linear programming. IEEE Trans. Info Theory, 51(12):4203-4215, December 2005.

[16] E. Candes and T. Tao. The Dantzig selector: Statistical estimation when $p$ is much larger than $n$. Annals of Statistics, 35(6):2313-2351, 2007.

[17] E. J. Candès and B. Recht. Exact matrix completion via convex optimization. Found. Comput. Math., 9(6):717-772, 2009.

[18] E. J. Candes, Y. Ma X. Li, and J. Wright. Stable principal component pursuit. In International Symposium on Information Theory, June 2010.

[19] V. Chandrasekaran, S. Sanghavi, P. A. Parrilo, and A. S. Willsky. Rank-sparsity incoherence for matrix decomposition. Technical report, MIT, June 2009. Available at arXiv:0906.2220v1.

[20] S. Chen, D. L. Donoho, and M. A. Saunders. Atomic decomposition by basis pursuit. SIAM J. Sci. Computing, 20(1):33-61, 1998.

[21] D. L. Donoho. Compressed sensing. IEEE Trans. Info. Theory, 52(4):1289-1306, April 2006.

[22] D. L. Donoho and J. M. Tanner. Neighborliness of randomly-projected simplices in high dimensions. Proceedings of the National Academy of Sciences, 102(27):9452-9457, 2005.

[23] M. Fazel. Matrix Rank Minimization with Applications. PhD thesis, Stanford, 2002. Available online: http://faculty.washington.edu/mfazel/thesis-final.pdf.

[24] V. L. Girko. Statistical analysis of observations of increasing dimension. Kluwer Academic, New York, 1995.

[25] E. Greenshtein and Y. Ritov. Persistency in high dimensional linear predictor-selection and the virtue of over-parametrization. Bernoulli, 10:971-988, 2004.

[26] D. Hsu, S. M. Kakade, and T. Zhang. Robust matrix decomposition with sparse corruptions. Technical report, Univ. Pennsylvania, November 2010.

[27] H. Hu, C. Caramanis, and S. Sanghavi. Robust PCA via outlier pursuit. Technical report, UT Austin, 2010.

[28] J. Huang and T. Zhang. The benefit of group sparsity. The Annals of Statistics, 38(4):1978-

imsart-sts ver. 2012/04/10 file: B_BaseFileStatSci.tex date: May 25, 2012 
2004, 2010.

[29] L. Jacob, G. Obozinski, and J. P. Vert. Group Lasso with Overlap and Graph Lasso. In International Conference on Machine Learning (ICML), pages 433-440, 2009.

[30] R. Jenatton, J. Mairal, G. Obozinski, and F. Bach. Proximal methods for hierarchical sparse coding. Technical report, HAL-Inria, 2010. available at inria-00516723.

[31] S. M. Kakade, O. Shamir, K. Sridharan, and A. Tewari. Learning exponential families in high-dimensions: Strong convexity and sparsity. In AISTATS, 2010.

[32] N. El Karoui. Operator norm consistent estimation of large-dimensional sparse covariance matrices. Annals of Statistics, 36(6):2717-2756, 2008.

[33] R. H. Keshavan, A. Montanari, and S. Oh. Matrix completion from noisy entries. Technical report, Stanford, June 2009. Preprint available at http://arxiv.org/abs/0906.2027v1.

[34] Y. Kim, J. Kim, and Y. Kim. Blockwise sparse regression. Statistica Sinica, 16(2), 2006.

[35] V. Koltchinskii and M. Yuan. Sparse recovery in large ensembles of kernel machines. In Proceedings of COLT, 2008.

[36] V. Koltchinskii and M. Yuan. Sparsity in multiple kernel learning. Annals of Statistics, 38:3660-3695, 2010.

[37] C. Lam and J. Fan. Sparsistency and rates of convergence in large covariance matrix estimation. Annals of Statistics, 37:4254-4278, 2009.

[38] D. Landgrebe. Hyperspectral image data analsysis as a high-dimensional signal processing problem. IEEE Signal Processing Magazine, 19(1):17-28, January 2008.

[39] M. Ledoux and M. Talagrand. Probability in Banach Spaces: Isoperimetry and Processes. Springer-Verlag, New York, NY, 1991.

[40] K. Lee and Y. Bresler. Guaranteed minimum rank approximation from linear observations by nuclear norm minimization with an ellipsoidal constraint. Technical report, UIUC, 2009. Available at arXiv:0903.4742.

[41] Z. Liu and L. Vandenberghe. Interior-point method for nuclear norm optimization with application to system identification. SIAM Journal on Matrix Analysis and Applications, 31(3):1235-1256, 2009.

[42] K. Lounici, M. Pontil, A. B. Tsybakov, and S. van de Geer. Taking advantage of sparsity in multi-task learning. Technical Report arXiv:0903.1468, ETH Zurich, March 2009.

[43] M. Lustig, D. Donoho, J. Santos, and J. Pauly. Compressed sensing MRI. IEEE Signal Processing Magazine, 27:72-82, March 2008.

[44] M. McCoy and J. Tropp. Two Proposals for Robust PCA using Semidefinite Programming. Technical report, California Institute of Technology, 2010.

[45] M. L. Mehta. Random matrices. Academic Press, New York, NY, 1991.

[46] L. Meier, S. van de Geer, and P. Buhlmann. High-dimensional additive modeling. Annals of Statistics, 37:3779-3821, 2009.

[47] N. Meinshausen. A note on the Lasso for graphical Gaussian model selection. Statistics and Probability Letters, 78(7):880-884, 2008.

[48] N. Meinshausen and P. Bühlmann. High-dimensional graphs and variable selection with the Lasso. Annals of Statistics, 34:1436-1462, 2006.

[49] N. Meinshausen and B. Yu. Lasso-type recovery of sparse representations for highdimensional data. Annals of Statistics, 37(1):246-270, 2009.

[50] Y. Nardi and A. Rinaldo. On the asymptotic properties of the group lasso estimator for linear models. Electronic Journal of Statistics, 2:605-633, 2008.

[51] S. Negahban, P. Ravikumar, M. J. Wainwright, and B. Yu. A unified framework for highdimensional analysis of $M$-estimators with decomposable regularizers. In NIPS Conference, 2009.

[52] S. Negahban, P. Ravikumar, M. J. Wainwright, and B. Yu. Supplement to "a unified framework for high-dimensional analysis of $M$-estimators with decomposable regularizers". 2012.

[53] S. Negahban and M. J. Wainwright. Estimation of (near) low-rank matrices with noise and high-dimensional scaling. Annals of Statistics, 39(2):1069-1097, 2011.

[54] S. Negahban and M. J. Wainwright. Simultaneous support recovery in high-dimensional regression: Benefits and perils of $\ell_{1, \infty}$-regularization. IEEE Transactions on Information Theory, 57(6):3481-3863, June 2011.

[55] S. Negahban and M. J. Wainwright. Restricted strong convexity and (weighted) matrix completion: Optimal bounds with noise. Journal of Machine Learning Research, 2012. Originally posted as arxiv:1009.2118. 
[56] G. Obozinski, M. J. Wainwright, and M. I. Jordan. Union support recovery in highdimensional multivariate regression. Annals of Statistics, 39(1):1-47, January 2011.

[57] L. A. Pastur. On the spectrum of random matrices. Theoretical and Mathematical Physics, 10:67-74, 1972 .

[58] G. Raskutti, M. J. Wainwright, and B. Yu. Restricted eigenvalue conditions for correlated Gaussian designs. Journal of Machine Learning Research, 11:2241-2259, August 2010.

[59] G. Raskutti, M. J. Wainwright, and B. Yu. Minimax rates of estimation for highdimensional linear regression over $\ell_{q}$-balls. IEEE Trans. Information Theory, 57(10):69766994, October 2011.

[60] G. Raskutti, M. J. Wainwright, and B. Yu. Minimax-optimal rates for sparse additive models over kernel classes via convex programming. Journal of Machine Learning Research, 12:389-427, March 2012.

[61] P. Ravikumar, H. Liu, J. Lafferty, and L. Wasserman. SpAM: sparse additive models. Journal of the Royal Statistical Society, Series B, 71(5):1009-1030, 2009.

[62] P. Ravikumar, M. J. Wainwright, and J. Lafferty. High-dimensional Ising model selection using $\ell_{1}$-regularized logistic regression. Annals of Statistics, 38(3):1287-1319, 2010.

[63] P. Ravikumar, M. J. Wainwright, G. Raskutti, and B. Yu. High-dimensional covariance estimation by minimizing $\ell_{1}$-penalized log-determinant divergence. Electron. J. Statist., 5:935-980, 2011.

[64] B. Recht. A simpler approach to matrix completion. Journal of Machine Learning Research, 12:3413-3430, 2011.

[65] B. Recht, M. Fazel, and P. Parrilo. Guaranteed minimum-rank solutions of linear matrix equations via nuclear norm minimization. SIAM Review, 52(3):471-501, 2010.

[66] A. Rohde and A. Tsybakov. Estimation of high-dimensional low-rank matrices. Annals of Statistics, 39(2):887-930, 2011.

[67] A. J. Rothman, P. J. Bickel, E. Levina, and J. Zhu. Sparse permutation invariant covariance estimation. Electronic Journal of Statistics, 2:494-515, 2008.

[68] M. Rudelson and S. Zhou. Reconstruction from anisotropic random measurements. Technical report, University of Michigan, July 2011.

[69] M. Stojnic, F. Parvaresh, and B. Hassibi. On the reconstruction of block-sparse signals with an optimal number of measurements. IEEE Transactions on Signal Processing, 57(8):30753085, 2009.

[70] R. Tibshirani. Regression shrinkage and selection via the lasso. Journal of the Royal Statistical Society, Series B, 58(1):267-288, 1996.

[71] R. Tibshirani, M. Saunders, S. Rosset, J. Zhu, and K. Knight. Sparsity and smoothness via the fused Lasso. J. R. Statistical Soc. B, 67:91-108, 2005.

[72] J. A. Tropp, A. C. Gilbert, and M. J. Strauss. Algorithms for simultaneous sparse approximation. Signal Processing, 86:572-602, April 2006. Special issue on "Sparse approximations in signal and image processing".

[73] B. Turlach, W.N. Venables, and S.J. Wright. Simultaneous variable selection. Technometrics, 27:349-363, 2005.

[74] S. van de Geer and P. Buhlmann. On the conditions used to prove oracle results for the lasso. Electronic Journal of Statistics, 3:1360-1392, 2009.

[75] S. A. van de Geer. High-dimensional generalized linear models and the lasso. The Annals of Statistics, 36:614-645, 2008.

[76] M. J. Wainwright. Information-theoretic bounds on sparsity recovery in the highdimensional and noisy setting. IEEE Trans. Info. Theory, 55:5728-5741, December 2009.

[77] M. J. Wainwright. Sharp thresholds for high-dimensional and noisy sparsity recovery using $\ell_{1}$-constrained quadratic programming (Lasso). IEEE Trans. Information Theory, 55:21832202, May 2009.

[78] E. Wigner. Characteristic vectors of bordered matrices with infinite dimensions. Annals of Mathematics, 62:548-564, 1955.

[79] M. Yuan, A. Ekici, Z. Lu, and R. Monteiro. Dimension reduction and coefficient estimation in multivariate linear regression. Journal Of The Royal Statistical Society Series B, 69(3):329-346, 2007.

[80] M. Yuan and Y. Lin. Model selection and estimation in regression with grouped variables. Journal of the Royal Statistical Society B, 1(68):49, 2006.

[81] C. H. Zhang and J. Huang. The sparsity and bias of the lasso selection in high-dimensional linear regression. Annals of Statistics, 36(4):1567-1594, 2008.

imsart-sts ver. 2012/04/10 file: B_BaseFileStatSci.tex date: May 25, 2012 
[82] P. Zhao, G. Rocha, and B. Yu. Grouped and hierarchical model selection through composite absolute penalties. Annals of Statistics, 37(6A):3468-3497, 2009.

[83] P. Zhao and B. Yu. On model selection consistency of Lasso. Journal of Machine Learning Research, 7:2541-2567, 2006.

[84] S. Zhou, J. Lafferty, and L. Wasserman. Time-varying undirected graphs. In 21st Annual Conference on Learning Theory (COLT), Helsinki, Finland, July 2008. 